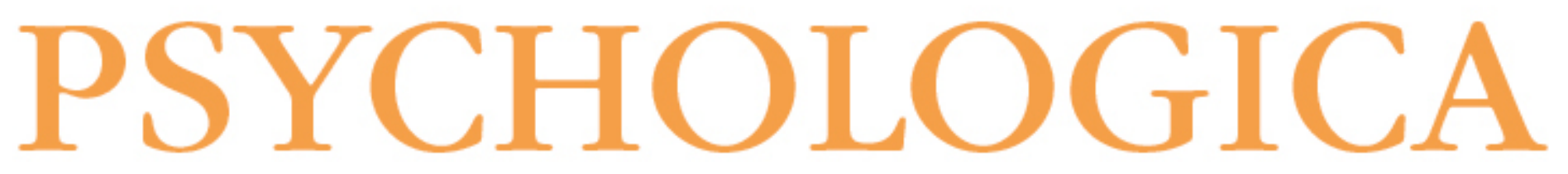

\title{
Estudo das propriedades psicométricas da Perceived Stress Scale (PSS) na população portuguesa
}

Autor(es): $\quad$ Trigo, Miguel; Canudo, Noélia; Branco, Fernando; Silva, Danilo

Publicado por: Imprensa da Universidade de Coimbra

URL

persistente: URI:http://hdl.handle.net/10316.2/5540

DOI: $\quad$ DOI:http://dx.doi.org/10.14195/1647-8606_53_17

Accessed : $\quad$ 26-Apr-2023 15:44:50

A navegação consulta e descarregamento dos títulos inseridos nas Bibliotecas Digitais UC Digitalis, UC Pombalina e UC Impactum, pressupõem a aceitação plena e sem reservas dos Termos e Condições de Uso destas Bibliotecas Digitais, disponíveis em https://digitalis.uc.pt/pt-pt/termos.

Conforme exposto nos referidos Termos e Condições de Uso, o descarregamento de títulos de acesso restrito requer uma licença válida de autorização devendo o utilizador aceder ao(s) documento(s) a partir de um endereço de IP da instituição detentora da supramencionada licença.

Ao utilizador é apenas permitido o descarregamento para uso pessoal, pelo que o emprego do(s) título(s) descarregado(s) para outro fim, designadamente comercial, carece de autorização do respetivo autor ou editor da obra.

Na medida em que todas as obras da UC Digitalis se encontram protegidas pelo Código do Direito de Autor e Direitos Conexos e demais legislação aplicável, toda a cópia, parcial ou total, deste documento, nos casos em que é legalmente admitida, deverá conter ou fazer-se acompanhar por este aviso. 
NÚMERO 53

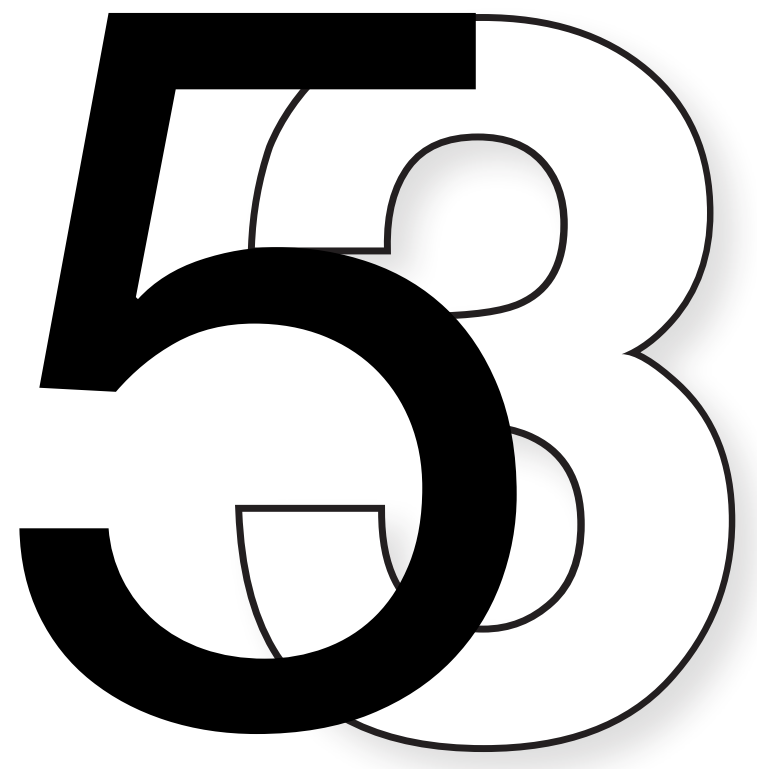

IMPRENSA DA UNIVERSIDADE DE COIMBRA

FACULDADE DE PSICOLOGIA E DE CIÊNCIAS DA EDUCAÇÃO DA UNIVERSIDADE DE COIMBRA 


\title{
Estudo das propriedades psicométricas da Perceived Stress Scale (PSS) na população portuguesa
}

\author{
Miguel Trigo', Noélia Canudo², Fernando Branco³ \& Danilo Silva4
}

Objectivo: Estudar as propriedades psicométricas da versão portuguesa da Perceived Stress Scale-10 (PSS-10).

Amostra: Cinco amostras da população geral $(n=762)$ e cinco amostras de pessoas com doença física ou perturbação ansiosa $(n=201)$.

Medidas: Dados socio-demográficos, hábito tabágico, Beck Depression Inventory ${ }^{\circ}$ $\left(\mathrm{BDI}^{\circ}\right)$, PSS-10, State-Trait Anxiety Inventory ${ }^{\circ}\left(\mathrm{STAl}^{\circ}-\mathrm{Y}_{2}\right)$ e Smoking Abstinence Self-Efficacy (SASE-12).

Resultados: Pela análise em componentes principais, reteve-se um factor que explica 47,4\% da variância. O alfa de Cronbach foi de o,874 e encontraram-se diferenças significativas na percepção do stresse, em diversos estratos da população. Existem correlações moderadas entre a PSS-10, o BDI ${ }^{\circ}$ e o STAI ${ }^{\circ}$-Y2 e ausência de associação com o SASE-12. Considera-se indicador de patologia pontuações brutas acima do percentil 80.

Conclusões: A PSS-10 apresenta boas qualidades psicométricas. A sua aplicação e cotação é simples, constituindo um instrumento válido para avaliar o stresse, em contextos de doença física ou condições psicopatológicas.

PALAVRAS-CHAVE: Perceived Stress Scale. Escala do Stresse Percepcionado. Stresse. Propriedades psicométricas.

\section{Introdução}

A experimentação científica sobre os mecanismos adaptativos e homeostáticos dos organismos vivos remonta ao século XIX, com os trabalhos pioneiros de Bernard (1879, 1978), de Meyer (1930) e de Canon (1939). Gradualmente proliferaram várias

\footnotetext{
1 Doutor em Psicologia Clínica. Especialista em Psicologia Clínica no Centro Hospitalar Psiquiátrico de Lisboa (CHPL), Unidade Comunitária de Cuidados Psiquiátricos de Odivelas (UCCPO) - miguel. trigo7o@gmail.com

2 Especialista em Psicologia Clínica. CHPL, UCCPO.

3 Professor Auxiliar convidado no Departamento de Psicologia da Universidade Lusófona de Humanidades e Tecnologias.

4 Professor Catedrático aposentado da Faculdade de Psicologia, Universidade de Lisboa.
} 
investigações na área do stresse, com a perspectiva biologicista a centrar-se nas respostas psicofisiológicas, a psicologista a focar-se na forma como o indivíduo avalia as pressões e a perspectiva ambientalista a enfatizar as exigências externas (stressor). Seriam, porém, os contributos, por um lado, de Selye (1936) com as noções de Síndroma Geral de Adaptação (General Adaptation Syndrome) e de agente indutor stresse (stressor) e, por outro lado, os avanços trazidos por Lazarus e Folkman (1984; Lazarus, 1991) com as noções de avaliação / percepção do stresse e estratégias de confronto (coping), que colocariam em definitivo os estudos sobre o stresse na charneira dos saberes biomédicos e psicossociais. É com base nestes contributos que o stresse deixa de ser considerado um estímulo (stressor), para passar a ser entendido como o resultado da exposição ao estímulo (Guillet \& Hermand, 2006). Pela dificuldade em delimitar o conceito stresse (Breznitz \& Goldberg, 1993), torna-se útil, pois, defini-lo como uma resposta individual, global e sistémica (corpo-mente) perante exigências ou estímulos indutores de pressão (Quick, Quick \& Gavin, 2000).

Associado às investigações sobre a natureza do stresse e o conhecimento das suas implicações para a saúde humana, tornou inevitável desenvolver vários métodos e técnicas de avaliação. A diversidade dos instrumentos de relato pessoal é hoje tão grande que podem-se agrupar em três grandes categorias (Guillet \& Hermand, 2006) $)^{5}$

- Escalas de acontecimentos de vida major. Inicialmente inspirados nos trabalhos de Meyer (1930), estas escalas desenvolveram-se em torno do estudo sobre as implicações da exposição a acontecimentos de vida positivos ou negativos (ex. morte do cônjuge, divórcio, casamento, Natal, férias). Os acontecimentos são aqui considerados como o elo de ligação entre o stresse e a doença psicossomática. O objectivo é enfatizar o papel dos factores psicossociais na etiopatogénese da doença, preconizando-se que os acontecimentos vitais aversivos têm uma natureza cumulativa que provoca a diminuição da resiliência e, por inerência, da saúde.

- Avaliação de acontecimentos de vida minor. Lazarus (1999) critica as escalas de acontecimentos de vida major por não valorizarem detalhes intrínsecos às situações ou às informações ligadas aos eventos, negligenciando quer o significado atribuído aos pequenos incidentes quer o facto de os eventos major poderem ser constituídos por uma colecção de incidentes menores. Na linha do que Holmes e Rahe (1967) haviam preconizado, também aqui

5 Outras classificações das medidas de stresse podem-se encontrar nos trabalhos de Derogatis e Coons (1993), no qual se distinguem três tipos de escalas (medidas orientadas para o estímulo, medidas orientadas para a resposta, medidas orientadas para a interacção) ou no texto de Quick, Quick e Gavin (2000), onde surgem quatro grupos de instrumentos (exigências ambientais e stressores, resposta saudável e normal ao stresse, modificadores da resposta ao stresse, distress e tensão psicológica, comportamental e biomédica) 
é aceite a influência das experiências emocionais positivas, neste caso pela sua acção na compensação dos efeitos deletérios provocados pela exposição a eventos negativos.

- Avaliação do stresse percepcionado. Cohen, Kamarck e Mermelstein (1983) colocam a ênfase no stresse percebido, em oposição ao stresse objectivo, considerando que este tipo de medida, mais directa, constitui um melhor preditor de saúde. Os estímulos de stresse, por si só, são insuficientes para provocar doença, pois o seu potencial patogénico resulta da transacção entre a pessoa e o ambiente. Deixam de ser os acontecimentos a determinar as respostas, para se valorizar a avaliação global que o indivíduo faz da situação. Assim, a percepção de stresse ocorrerá apenas quando uma situação é avaliada como ameaçadora ou exigente e o sujeito dispõe de poucos recursos para lidar com esse desafio.

O valor da concepção baseada no stresse percepcionado, juntamente com a necessidade de desenvolver estudos neste domínio e o interesse suscitado pela Perceived Stress Scale - PSS (Cohen, Kamarck \& Mermelstein, 1983), têm justificado a publicação de várias versões desta escala. Refiram-se, a este propósito, os trabaIhos desenvolvidos no Brasil (Luft et al., 2007), no México (Ramírez \& Hernández, 2007), no Japão (Mimura \& Griffiths, 2004; Shigetoshi et al., 2002; Sumi, 2006), na Hungria (Adrienne \& Barna, 2006) ou na Espanha (Remor, 2006). Semelhante interesse está também na base de investigações em diversas populações, designadamente de estudantes universitários (Roberti, Harrington \& Storch, 2006), doentes com asma (Sharp et al., 2007), pessoas com cancro (Golden-Kreutz et al., 2004), vírus da imunodeficiência humana (Remor \& Carrobles, 2001) ou depressão (Hewitt, Flett \& Mosher, 1992). A nível nacional, porém, não se conhecem estudos sobre as propriedades psicométricas da PSS. Algumas das mais recentes colectâneas sobre instrumentos validados para a população portuguesa (Almeida et al., 2008; Gonçalves, 2006; Ribeiro, 2007; Simões et al., 2007), apesar de incluírem várias escalas para avaliação do stresse, particularmente no trabalho de Ribeiro (2007), nenhuma se refere ao stresse percepcionado. Este facto parece justificar a pertinência do presente trabalho.

\section{Instrumento}

A PSS (Cohen, Kamarck \& Mermelstein, 1983) é um dos instrumentos de relato pessoal mais utilizados ${ }^{6}$ na avaliação global do stresse, permitindo determinar

\footnotetext{
6 Pode-se ler no endereço electrónico da Mind Garden, Inc. ${ }^{\circledR}$ (http://mindgarden.com/products/pss.htm acedido em 07/01/2008) que a PSS é "o instrumento psicológico mais frequentemente usado para medir o stresse percepcionado" (tradução livre).
} 
até que ponto os acontecimentos de vida são percebidos como indutores do stresse, em consequência do seu carácter imprevisivel, incontrolável ou excessivo. Considerando que o stresse percepcionado é influenciado por eventos quotidianos, relações interpessoais e alterações na disposição pessoal, o valor preditivo da PSS pode diminuir acentuadamente nas quatro a seis semanas após a sua aplicação. Assim, a instrução da PSS refere-se à frequência com que um contexto geral de vida ocorreu durante o último mês, não especificando uma situação particular (ex. item 3 - "No último mês, com que frequência se sentiu nervoso e em stresse?"). Existem três versões da PSS, uma mais longa com catorze itens (PSS-14), outra com dez itens (PSS-10) e uma forma reduzida com quatro itens (PSS-4), caracterizando-se todas pela facilidade de aplicação e cotação (op cit.; Cohen \& Williamson, 1988). A PSS-10 inclui os itens 1 a 3 , 6 a 11 e 14, enquanto a PSS-4 é composta pelos itens 2, 6, 7 e 14. As respostas são assinaladas mediante uma escala de Likert com cinco pontos (o - nunca, 1 - quase nunca, 2 - algumas vezes, 3 - frequentemente e 4 - muito frequente) e os resultados totais oscilam entre 0-56 (PSS-14), 0-40 (PSS-10) e 0-16 (PSS-4). Para calcular a pontuação final é necessário ter em consideração que vários itens se referem a situações positivas, sendo necessário inverter a cotação das respostas aos itens 4, 5, 6, 7, 9, 10 e 13, na PSS-14, aos itens 4, 5, 7 e 8, na PSS-10 e aos itens 2 e 3 , na PSS-4 (ver Quadro I).

Quadro I. Composição das escalas e itens cotados de forma inversa em diferentes versões da PSS.

\begin{tabular}{lcccccccccccccc}
\hline Versão & 1 & 110 & \multicolumn{11}{c}{${ }^{\circ}$ do item } \\
\hline PSS-14 & 1 & 2 & 3 & 4 & 5 & 6 & 7 & 8 & 9 & 10 & 11 & 12 & 13 & 14 \\
PSS-10 & 1 & 2 & 3 & & & 4 & 5 & 6 & 7 & 8 & 9 & & & 10 \\
PSS-4 & & 1 & & & & 2 & 3 & & & & & & & 4 \\
\hline
\end{tabular}

Os números a negrito e itálico são cotados de forma inversa.

Entre os principais estudos desenvolvidos por Cohen e associados (1983, 1988), relativos aos valores normativos e qualidades psicométricas da PSS, destaca-se o trabalho datado de 1988, que envolveu uma amostra estratificada de 2387 cidadãos norte-americanos. As pontuações médias obtidas pelo total de participantes, na PSS-14, PSS-10 e PSS-4, foram, respectivamente, 19,62 ( $D P=7,49), 13,02(D P=6,35)$ e 4,49 $(D P=2,96)$. Registaram-se niveis de stresse mais elevados nas mulheres do que nos homens e observou-se uma correlação negativa fraca, mas significativa, entre as pontuações obtidas na PSS e a idade, sugerindo que a percepção de stresse tende a declinar à medida que a idade aumenta. Os caucasianos referiram menos stresse do que outros grupos étnicos, embora esta diferença só seja estatisticamente significativa quando comparada com os negros. As pontuações 
na PSS também diminuíram à medida que o nível educacional era mais elevado. Inversamente, com o aumento da dimensão do agregado familiar, tornou-se mais elevada a percepção de stresse.

O estudo da precisão das três versões da PSS baseou-se no cálculo dos coeficientes alfa de Cronbach, os quais apresentam respectivamente os seguintes valores: 0,75, 0,78 e o,60. Através da análise factorial à PSS-14, usando o método dos componentes principais com rotação Varimax, identificaram-se dois factores. O primeiro foi composto por dez itens com saturações factoriais superiores ou iguais a 0,48 e o segundo foi formado pelos itens 4, 5, 12 e 13. Enquanto no factor I (25,9\% da variância) saturaram mais as perguntas relativas a situações negativas, no factor II (15,7\% da variância) saturaram mais os itens relativos a situações positivas. No seu conjunto, os dois factores explicaram $41,6 \%$ da variância total. A PSS-10 resultou precisamente da eliminação dos quatro itens com menor saturação no factor I (4, 5, 12 e 13), procedimento que introduziu uma melhoria ligeira, quer na variância explicada (48,9\%), quer na consistência interna $(\alpha=0,78)$. No que diz respeito à PSS-4, apenas foi extraído um factor que explicou $45,6 \%$ da variância. Um estudo que avaliou o funcionamento diferencial dos itens da PSS-10 (Cole, 1999), revelou que nos itens 3, 6, 7, 8 e 10 as mulheres apresentavam pontuações mais elevadas. Nos itens 3 e 4 , são os caucasianos a pontuar mais alto, enquanto que nos itens 3 e 9 os participantes mais escolarizados referem mais stresse e, nos itens 4 e 8 , as pessoas com menos anos de escolaridade obtêm pontuações mais elevadas. De uma forma geral, porém, as pontuações obtidas nos diferentes itens da PSS-10 mantiveram-se estáveis, o que sugere tratar-se de uma medida unidimensional.

Os dados relativos à validade convergente (Cohen \& Williamson, 1988) indicam que as pontuações da PSS se associam a outras medidas e variáveis, designadamente de stresse percepcionado, sintomas emocionais, doença física, redução dos períodos de sono ou quantidade no consumo de álcool e outras drogas. Menos significativa foi a relação entre a PSS e o sedentarismo ou o tabagismo. Neste último caso, porém, os fumadores parecem apresentar níveis mais elevados de stresse do que os seus pares não fumadores. Quanto à validade divergente importa referir que a PSS se relaciona inversamente com indicadores de satisfação com a vida.

As versões adaptadas para vários países, revelam, igualmente, valores de consistência interna bastante satisfatórios, geralmente superiores aos apresentados pela versão original (ver Quadro II). No caso da PSS-4, os coeficientes alfa de Cronbach oscilam (mín. e máx.) entre o,6o (Cohen \& Williamson, 1988) e o,85 (Adrienne \& Barna, 2006), na PSS-10 entre 0,71 (Sumi, 2006) e 0,89 (Roberti, Harrington \& Storch, 2006) e na PSS-14 entre 0,67 (Remor \& Carrobles, 2001) e o,89 (Shigetoshi 
et al., 2002). A solução de dois factores também tem sido corroborada em vários trabalhos (Golden-Kreutz et al., 2004; Hewitt, Flett \& Mosher, 1992; Mimura \& Griffiths, 2004; Ramírez \& Hernández, 2007; Roberti, Harrington \& Storch, 2006), identificando-se, por um lado, os itens que se referem a situações positivas e de auto-eficácia (ex. item 4 - "No último mês, com que frequência sentiu confiança em si?") e, por outro lado, os que envolvem eventos negativos e sensação de impotência (ex. item 2 - "No último mês, foi incapaz de controlar as coisas?").

Quadro II. Resumo dos alfas de Cronbach usando a Perceived Stress Scale em diferentes populações.

\begin{tabular}{|c|c|c|c|c|}
\hline Versão / país & Autor & PSS-4 & PSS-10 & PSS-14 \\
\hline Espanha & Remor (2006) & - & 0,82 & 0,81 \\
\hline Espanha* & Remor e Carrobles (2001) & - & - & 0,67 \\
\hline Brasil (idosos) & Luft et al. (2007) & - & 0,83 & 0,82 \\
\hline EUA & Cohen e Williamson (1988) & 0,60 & 0,78 & 0,75 \\
\hline EUA (ens. secund.) & Roberti, Harrington e Storch (2006) & - & 0,89 & - \\
\hline \multirow[t]{2}{*}{ Hungria } & Adrienne e Barna (2006) & 0,85 & 0,88 & 0,79 \\
\hline & Mimura e Griffiths, 2004 & - & - & 0,81 \\
\hline \multirow[t]{2}{*}{ Japão } & Shigetoshi et al., (2002) & - & - & $0,82^{* *}-0,89^{* * *}$ \\
\hline & Sumi (2006) & - & 0,71 & 0,76 \\
\hline México & Ramírez e Hernández (2007) & - & - & 0,83 \\
\hline
\end{tabular}

'População com Vírus da Imunodeficiência Humana; “População com sintomas físicos; “"População com sintomas psíquicos.

\section{Objectivos}

- Estudar a estrutura factorial da versão de dez itens da Perceived Stress Scale (PSS-10), em amostras da população geral portuguesa (amostras $1-5 ; n=762)$.

- Estudar a consistência interna da PSS-10, num grupo de adultos jovens e adultos da população geral portuguesa.

- Calcular valores normativos (média e desvio-padrão) na PSS-10, para ambos os sexos, em cinco amostras da população geral e em cinco amostras de pessoas com doença (amostras 6 - 10; $n=201$ ), designadamente doença física crónica (amostras 6 e 7), hipertensão arterial, dislipidémia ou diabetes (amostra 8) e perturbações da ansiedade (amostras 9 e 10). 
- Estudar as correlações da PSS-10 com outros indicadores e medidas de saúde (dados socio-demográficos, hábitos tabágicos, depressão, avaliada pelo Beck Depression Inventory ${ }^{\circ}$, traço de ansiedade, avaliada pelo StateTrait Anxiety Inventory ${ }^{\circ}$ - Forma Y2, auto-eficácia, avaliada pelo Smoking Abstinence Self-Efficacy, versão de quatro itens da PSS e pacientes com perturbação ansiosa), em amostras da população portuguesa.

\subsection{Participantes}

O estudo das qualidades psicométricas da PSS-10 tem por base dez amostras não probabilísticas da população portuguesa, com residência no Distrito de Lisboa (ver Quadro III). As amostras da população geral (amostras 1 a 5 ) incluem 305 homens e 457 mulheres, com idades compreendidas entre os $17 \mathrm{e}$ os 73 anos $(M=33,4 ; D P=12,2)$. A idade média nos homens é 35,7 $(D P=12,3)$ e nas mulheres é 31,9 $(D P=11,9)$. Estas amostras foram recolhidas no Instituto Nacional de Engenharia e Tecnologia Industrial - Posto Médico (amostra 1), na Sãvida-Medicina Apoiada, S.A. - Grupo EDP (amostra 2), na FAPIL - Indústria de Escovaria, S.A. (amostra 3), numa Universidade (amostra 4) e na comunidade, através do método "bola de neve".

As amostras de participantes com doença (amostras 6 a 10) referem-se a pessoas com doença física crónica, designadamente doença das artérias coronárias - DAC (amostra 6), doença pulmonar obstrutiva crónica - DPOC (amostra 7), hipertensão arterial, dislipidémia ou diabetes - HDD (amostra 8) e doentes ansiosos (amostras 9 e 10). A amostra 6, tem 52 pacientes cardíacos, a amostra 7, tem 50 pessoas com DPOC, a amostra 8, tem 27 sujeitos com hipertensão arterial, dislipidémia ou diabetes e as amostras 9 e 10, têm 37 pacientes ansiosos. Nestas amostras (6 a 10), observou-se maior amplitude de idades do que nas amostras da população geral, oscilando entre os 17 e os 86 anos $(M=54,9 ; D P=17,1)$. Os locais de recolha foram o Hospital Militar Principal - Serviço de Cardiologia (amostra 6), o Hospital de Pulido Valente, S.A. - Hospital de Dia de Insuficientes Respiratórios (amostra 7), a Clínica de Santo António, S.A. (amostra 8), o Hospital Júlio de Matos (amostra 9) e o Hospital Miguel Bombarda (amostra 10). 
Quadro III. Distribuição das amostras por local de recolha e sexo.

\begin{tabular}{|c|c|c|c|c|c|}
\hline & \multirow{2}{*}{ Amostra } & \multirow{2}{*}{ Local } & \multicolumn{2}{|c|}{ Sexo } & \multirow{2}{*}{ Tota } \\
\hline & & & Homens & Mulheres & \\
\hline \multirow{6}{*}{ 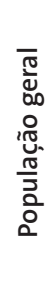 } & 1 & INETI & 77 & 152 & 229 \\
\hline & 2 & EDP & 49 & 21 & 70 \\
\hline & 3 & FAPIL & 7 & 33 & 40 \\
\hline & 4 & Univ. & 48 & 213 & 261 \\
\hline & 5 & Univ. - BN & 124 & 38 & 162 \\
\hline & Subtotal & & 305 & 457 & 762 \\
\hline \multirow{7}{*}{ 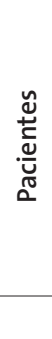 } & 6 & HMP & 41 & 11 & 52 \\
\hline & 7 & HPV & 44 & 6 & 50 \\
\hline & 8 & CLISA & 15 & 12 & 27 \\
\hline & 9 & HJM & 8 & 13 & 21 \\
\hline & 10 & $\mathrm{HMB}$ & 26 & 25 & 51 \\
\hline & Subtotal & & 134 & 67 & 201 \\
\hline & Total & & 439 & 524 & 963 \\
\hline
\end{tabular}

Legenda: CLISA = Clínica de Santo António, S.A.; EDP = Sãvida-Medicina Apoiada, S.A., Grupo EDP; FAPIL = Indústria de Escovaria, S.A.; HJM = Hospital Júlio de Matos; HMB = Hospital Miguel Bombarda; HMP = Hospital Militar Principal, Serviço de Cardiologia; HPV = Hospital de Pulido Valente, S.A., Hospital de Dia de Insuficientes Respiratórios; INETI = Instituto Nacional de Engenharia e Tecnologia Industrial, Posto Médico; Univ. = Universidade; Univ. - BN = Universidade - Bola de neve.

\subsection{Medidas e instrumentos}

- Dados socio-demográficos. Os participantes foram identificados quanto ao sexo, idade, estado civil, nacionalidade e com base na classificação de Graffar (1956) modificada, definiram-se quatro graus de escolaridade (> 12 anos, 10 - 12 anos, 8 - 9 anos, 4 - 6 anos) e cinco graus profissionais (ex. Director, Sub-director, Capataz, Polícia, Jornaleiro).

- Hábitos tabágicos. Definiram-se os hábitos tabágicos a partir da Entrevista Estruturada para Avaliação de Hábitos tabágicos (Trigo et al., 2007), através de questões sobre o estatuto de fumador (não fumador, ex-fumador e fumador), o número de cigarros fumados por dia e os anos de tabagismo.

- Diagnóstico de doença. Os quadros de doença física basearam-se no diagnóstico médico, segundo os critérios da décima versão da Classificação Internacional de Doenças (WHO, 2005). O diagnóstico das perturbações ansiosas foi estabelecido pelo psiquiatra ou pelo psicólogo, de acordo com o texto revisto da quarta edição do Manual de diagnóstico e estatísticas das doenças mentais (APA, 2002). 
- Beck Depression Inventory ${ }^{\circ}\left(B D I^{\circ}\right)$. O BDI ${ }^{\circ}$ (Beck et al., 1979; Trigo \& Silva, 2003a)7 é um inventário de relato pessoal que avalia a depressão, através de vinte e um itens. A resposta ao inventário faz-se por meio de uma escala de Guttman com quatro afirmações, cotadas entre o e 3 pontos. A pontuação final resulta da soma dos pontos obtidos em cada item, podendo variar entre o e 63. Através da análise dos componentes principais, com rotação Varimax e do estudo da consistência interna, excluíram-se os itens $1,2,4,5,6,8,11,12,16,18,19$ e 20 . Os nove itens restantes constituíram quatro componentes capazes de explicar $57,23 \%$ da variância. O valor do alfa de Cronbach foi o,66 e a correlação média inter-item de 0,19 (Trigo, 2007).

- Anxiety Trait Scale (Y2) do State-Trait Anxiety Inventory ${ }^{\circ}\left(\mathrm{STAl}^{\circ}\right)$. O STAI ${ }^{\circ}$ (Spielberger, 1983; Silva \& Correia, s/d) ${ }^{8}$ é um inventário de relato pessoal composto por duas medidas, uma que avalia o estado $\left(\mathrm{Y}_{1}\right)$ e a outra o traço (Y2) de ansiedade. No total existem quarenta itens que são respondidos recorrendo a uma escala de Likert com quatro pontos (1 - 4). As pontuações podem variar entre 20 e 80 pontos, em cada escala. 0 estudo das qualidades psicométricas da Anxiety Trait Scale do STAl ${ }^{\circ}$, justificou a eliminação dos itens $2,4,5,8,12,13,14$ e 15, tendo-se retido dois factores teoricamente interpretáveis. A variância explicada pelos doze itens foi de $56,74 \%$ e o valor do alfa situou-se nos 0,88, com uma correlação média inter-item de $r=0,38$ (Trigo, 2007).

- Smoking Abstinence Self-Efficacy (SASE-12). O SASE-12 (DiClemente, 1981; Trigo \& Silva, 2003b) é uma escala de preenchimento pessoal que avalia a capacidade dos fumadores resistirem à tentação de fumar em doze situações. A resposta é dada através de uma escala de Likert com sete pontos $(1-7)$. A cotação da escala é obtida pela soma das respostas em cada item, podendo variar entre 12 e 84 pontos. Considerando a especificidade do instrumento, a sua aplicação é limitada aos fumadores. Pela análise dos componentes principais, com rotação Varimax, extraíram-se dois componentes interpretáveis e obteve-se uma variância explicada de $56,71 \%$. O alfa de Cronbach foi de o,88 e as correlações médias inter-item de 0,38 (Trigo, 2007).

7 Tradução portuguesa copyright@ 2006 de Aaron T. Beck. Traduzido e reproduzido com permissão do editor. Harcourt Assessment, Inc. Todos os direitos reservados. Versão portuguesa preparada por Trigo e Silva (2003).

8 Tradução portuguesa copyright @ 1968, 1977 de Charles Spielberger. Traduzido e reproduzido com permissão do editor. Mind Garden, Inc. Todos os direitos reservados. Versão portuguesa preparada por Silva e Correia (s/d). Aplicação autorizada no âmbito dos estudos de adaptação efectuados por Danilo R. Silva (s/d). 


\subsection{Procedimento}

Considerando que a utilização da PSS para fins educativos ou de investigação está autorizada pelos autores da escala9 ${ }^{9}$, procedeu-se à sua tradução para a língua portuguesa (ver anexo) e respectiva retroversão. Simultaneamente, foi pedido o parecer de vários peritos na área da Psicologia e da saúde mental, de leitores de inglês e bilingues de língua portuguesa e inglesa. A tradução e preparação dos restantes materiais seguiu procedimentos semelhantes.

Numa fase experimental, fizeram-se aproximadamente vinte aplicações para identificar dificuldades na compreensão das instruções ou dos itens e seguidamente reproduziram-se em série os protocolos de resposta. Neste período, decorreu também o treino dos testadores e estabeleceu-se um esquema de supervisão. Como é sabido, a clareza na redacção dos itens e das instruções, a adequação das condições para aplicação de um instrumento e o treino dos entrevistadores, constituem procedimentos fundamentais na redução dos erros de medida (Nunnally \& Bernstein, 1994).

Em oito dos locais em que decorreu a recolha (INETI, EDP, FAPIL, HMP, HPV, CLISA, HJM e HMB), existia um gabinete médico-psicológico devidamente equipado para a realização de consultas. Nestas situações, houve um controlo adequado das condições de entrevista e aplicação dos questionários. A relação entre o participante e o entrevistador foi fácil de estabelecer, as instruções verbais foram claramente percebidas, os dias e horários de recolha coincidiram com a deslocação das pessoas à consulta médica, as salas estavam devidamente isoladas de ruídos ou distracções, a iluminação, ventilação, espaço e assento foram satisfatórios, sendo também garantida a privacidade e tranquilidade necessárias a este tipo de procedimentos (Anastasi, 1977). Após as sessões de trabalho os protocolos ficaram à guarda dos entrevistadores. Todos os participantes deram o seu consentimento informado, tendo-se garantido o anonimato, a confidencialidade, a adequação das técnicas utilizadas e a possibilidade de abandonar o estudo a qualquer momento.

As aplicações em grupo, efectuadas numa Universidade e os casos recolhidos através do método "bola de neve", foram menos padronizados. Na Universidade, as condições físicas e logísticas foram aquelas que servem ao funcionamento geral das aulas, tendo o processo de recolha sido adequado. A este propósito devemos referir que as aplicações colectivas podem dar mais uniformidade de condições do que os testes individuais, pois o papel do examinador é menor (Anastasi, 1977, p. 254). No que se refere à amostra angariada pelo método

\footnotetext{
9 Para aceder a esta informação, consultar o seguinte endereço: http://www.macses.ucsf.edu/Research/ Psychosocial/notebook/PSS10.html. Versão portuguesa preparada por Trigo e Silva (2003).
} 
"bola de neve" é, naturalmente, mais difícil avaliar até que ponto foram respeitados os requisitos inerentes à prática da avaliação psicológica. Aquilo que se garantiu foi que os testadores recebessem formação prévia e supervisão regular. Durante todo o percurso e atendendo ao nível de motivação destes colaboradores, foi correcta a interiorização que fizeram dos objectivos e procedimentos a adoptar.

\section{Estrutura factorial}

A análise da estrutura factorial da PSS-10 restringiu-se às amostras da população geral ( $n=758$ ), a fim de obter maior controlo sobre as variáveis de confundimento. Considerando que no estudo da consistência interna se irá proceder à categorização da idade em duas classes (17- 25 e $26-65$ anos de idade), retiraram-se quatro homens, por apresentarem idade superior a 65 anos. $O$ estrato masculino passou, assim, a incluir 301 elementos. Aferiu-se a qualidade das correlações e garantiu-se a aplicabilidade do modelo factorial, usando o teste Kaiser-Meyer-Olkin $(\mathrm{KMO}=0,908)^{10}$ e o teste de esfericidade de Bartlett $\left(\chi^{2}=2769,057 ; g l=45 ; p=0,000\right)$ que confirmaram a possibilidade de utilizar este procedimento estatístico.

Para estudar a estrutura factorial dos dez itens da PSS efectuou-se uma análise em componentes principais. O número de componentes a reter foi determinado pela regra de Kaiser (valores próprios > 1) e mantiveram-se os itens com saturação factorial superior a 0,50"1 (Nunnaly \& Bernstein, 1994). Deste modo, foi obtida uma solução unifactorial, capaz de explicar $47,4 \%$ da variância total. Este resultado é teoricamente interpretável, replicando a estrutura factorial original. Através da matriz de componentes (ver Quadro IV), observa-se que os vários itens apresentam saturações factoriais salientes, oscilando entre 0,789 e 0,578 , valores que se enquadraram nos critérios definidos $(r \geq 0,50)$. Estes dados justificam que os dez itens originais sejam mantidos na versão portuguesa da PSS-10.

10 Critérios para qualificação dos valores de KMO (Pestana \& Gageiro, 2005, p. 491): 1 - o,9 (muito bom), 0,8 - 0,9 (bom), 0,7 - 0,8 (médio), 0,6 - 0,7 (razoável), 0,5 - 0,6 (mau), < 0,5 (inaceitável).

11 Critério para qualificação da saturação factorial (Nunnally \& Bernstein, 1994, p. 466): $r \geq 0$,30, preferencialmente $r \geq 0,50$. 
Quadro IV. Matriz de componentes principais dos itens da PSS-10.

\begin{tabular}{lc}
\hline Itens & Componente 1 \\
\hline PSS10 & 0,789 \\
PSS8 & 0,758 \\
PSS5 & 0,740 \\
PSS2 & 0,735 \\
PSS9 & 0,709 \\
PSS3 & 0,697 \\
PSS6 & 0,628 \\
PSS1 & 0,611 \\
PSS4 & 0,601 \\
PSS7 & 0,578 \\
\hline
\end{tabular}

\section{Consistência interna}

Para o estudo da consistência interna, definiu-se que um bom coeficiente alfa de Cronbach seria $\geq 0,80^{12}$, que as correlações item-total deveriam ter valores $\geq 0,30 \mathrm{e}$ que os níveis de correlação média inter-item estariam compreendidos entre 0,20 e 0,40 (Nunnally \& Bernstein, 1994; Pestana \& Gageiro, 2005). Com a finalidade de avaliar a existência de diferenças na consistência interna, estratificaram-se as amostras da população geral por sexo e em duas categorias etárias que incluíram adultos jovens (17- 25 anos de idade; $M=22,1 ; D P=1,7$ ) e adultos ( $26-65 ; M$ $=42,1 ; D P=11,1)$. No grupo de adultos jovens do sexo masculino, as correlações item-total corrigido situaram-se abaixo dos valores desejáveis, relativamente aos itens PSS1 $(r=0,259)$ e PSS7 ( $r=0,235)$. Segundo Silva e Campos (1999), porém, existem autores que consideram aceitáveis valores mínimos de correlação até aos 0,20 (min. $r=0,20 /$ máx. $r=0,80$ ). Quanto aos valores de correlação média inter-item, nos homens, situaram-se dentro da amplitude definida $(r=0,340)$ e, nas mulheres, o limite superior foi ligeiramente ultrapassado $(r=0,443)$. Incluindo ambos os sexos, as correlações item-total corrigido oscilaram entre $r=0,235 \mathrm{e}$ $r=0,739$, o que se pode considerar adequado (ver Quadro V).

Em relação aos coeficientes alfa, os valores obtidos pelo grupo de homens $(\alpha=0,84)$ e mulheres $(\alpha=0,89)$ foram ambos bons, embora ligeiramente mais elevados nas mulheres. $\mathrm{O}$ alfa de Cronbach apenas poderia ser melhorado no sexo masculino, através da eliminação dos itens PSS1 e PSS7, passando a obter-se alfas de 0,85,

12 Critérios para qualificação do coeficiente alfa de Cronbach (Pestana \& Gageiro, 2005, p. 526): > 0,9 (muito bom), o,8 - o,9 (bom), 0,7 - o,8 (razoável), o,6 - o,7 (fraco) e < o,6 (inadmissível). 
em vez de 0,84. A este respeito deve-se notar, por um lado, que o acréscimo na consistência interna é diminuto e, por outro lado, que, para fins de investigação, os erros de medida acima de um alfa de o,80 são pouco significativos (Nunnally \& Bernstein, 1994). Existem, pois, indicações bastante positivas relativamente à consistência interna da PSS-10 no estrato dos jovens.

Quadro V. Correlação item-total corrigida e coeficiente alfa de Cronbach da PSS-10, por sexo, em jovens entre os 17 e 25 anos de idade.

\begin{tabular}{l|c|c|c|c}
\hline \multirow{2}{*}{ Item n. } & \multicolumn{2}{|c|}{ Homens $(\mathbf{n}=93)$} & \multicolumn{2}{c}{ Mulheres (n=198) } \\
\cline { 2 - 5 } & $\begin{array}{c}\text { Correlação } \\
\text { item-total } \\
\text { corrigida }\end{array}$ & $\begin{array}{c}\text { Alfa de } \\
\text { Cronbach } \\
\text { eliminando o } \\
\text { item }\end{array}$ & $\begin{array}{c}\text { Correlação } \\
\text { item-total } \\
\text { corrigida }\end{array}$ & $\begin{array}{c}\text { Alfa de } \\
\text { Cronbach } \\
\text { eliminando o } \\
\text { item }\end{array}$ \\
\hline PSS1 & 0,259 & 0,849 & 0,518 & 0,884 \\
\hline PSS2 & 0,522 & 0,823 & 0,681 & 0,872 \\
\hline PSS3 & 0,697 & 0,805 & 0,571 & 0,880 \\
\hline PSS4 & 0,443 & 0,831 & 0,594 & 0,878 \\
\hline PSS5 & 0,601 & 0,816 & 0,730 & 0,870 \\
\hline PSS6 & 0,695 & 0,806 & 0,502 & 0,885 \\
\hline PSS7 & 0,235 & 0,847 & 0,557 & 0,881 \\
\hline PSS8 & 0,694 & 0,808 & 0,665 & 0,874 \\
\hline PSS9 & 0,483 & 0,827 & 0,683 & 0,872 \\
\hline PSS10 & 0,711 & 0,805 & 0,739 & 0,868 \\
\hline Correlação média & & 0,340 & & 0,443 \\
\hline inter-item & & & & 0,887 \\
\hline
\end{tabular}

Na faixa etária dos adultos (26 - 65 anos de idade), as correlações item-total corrigido foram satisfatórias, variando entre $r=0,392$ e $r=0,70$. Em relação aos valores de correlação média inter-item, ultrapassaram ligeiramente, nos homens, o limite superior $(r=0,416)$, enquanto, nas mulheres, situaram-se dentro da amplitude esperada $(r=0,354)$. Os coeficientes alfa foram bons e sensivelmente mais elevados nos homens $(\alpha=0,88)$ do que nas mulheres $(\alpha=0,85)$. No estrato masculino e feminino, não existiu indicação de itens a eliminar para aumentar o coeficiente alfa. Estes valores atestam a boa consistência interna da PSS-10, numa amostra composta por adultos portugueses (ver Quadro VI). 
Quadro VI. Correlação item-total corrigida e coeficiente alfa de Cronbach da PSS-10, por sexo, em adultos entre os 26 e 65 anos de idade.

\begin{tabular}{|c|c|c|c|c|}
\hline \multirow[b]{2}{*}{ Item n. ${ }^{\circ}$} & \multicolumn{2}{|c|}{ Homens $(n=202)$} & \multicolumn{2}{|c|}{ Mulheres $(n=252)$} \\
\hline & $\begin{array}{c}\text { Correlação } \\
\text { item-total } \\
\text { corrigida }\end{array}$ & $\begin{array}{c}\text { Alfa de } \\
\text { Cronbach } \\
\text { eliminando o } \\
\text { item }\end{array}$ & $\begin{array}{c}\text { Correlação } \\
\text { item-total } \\
\text { corrigida }\end{array}$ & $\begin{array}{c}\text { Alfa de } \\
\text { Cronbach } \\
\text { eliminando o } \\
\text { item }\end{array}$ \\
\hline PSS1 & 0,601 & 0,865 & 0,491 & 0,837 \\
\hline PSS2 & 0,661 & 0,860 & 0,628 & 0,824 \\
\hline $\mathrm{PSS}_{3}$ & 0,567 & 0,868 & 0,595 & 0,827 \\
\hline $\mathrm{PSS}_{4}$ & 0,456 & 0,875 & 0,453 & 0,839 \\
\hline $\mathrm{PSS}_{5}$ & 0,617 & 0,864 & 0,610 & 0,827 \\
\hline PSS6 & 0,595 & 0,865 & 0,393 & 0,846 \\
\hline PSS7 & 0,539 & 0,869 & 0,392 & 0,844 \\
\hline PSS8 & 0,642 & 0,862 & 0,642 & 0,823 \\
\hline PSS9 & 0,628 & 0,863 & 0,570 & 0,829 \\
\hline PSS10 & 0,700 & 0,857 & 0,676 & 0,819 \\
\hline $\begin{array}{l}\text { Correlação média } \\
\text { inter-item }\end{array}$ & \multicolumn{2}{|c|}{0,416} & \multicolumn{2}{|c|}{0,354} \\
\hline$\alpha$ & \multicolumn{2}{|c|}{0,877} & \multicolumn{2}{|c|}{0,846} \\
\hline
\end{tabular}

Dado não existirem diferenças significativas nos coeficientes alfa, entre as duas faixas etárias e os sexos, decidiu-se estudar a consistência interna da PSS-10 sem estratificação. Como resultado, aumentou a dimensão das amostras da população geral ( $n=745$ ), ficando muito acima das trezentas pessoas, sugeridas por Nunnally e Bernstein (1994) como número mínimo representativo para se efectuarem análises de itens. Com esta reorganização (ver Quadro VII), obtiveram-se correlações item-total corrigido que variaram entre $r=0,484$ (PSS7) e $r=0,712$ (PSS10), embora a correlação média inter-item tenha ultrapassado ligeiramente o limite superior $(r=0,410)$. O coeficiente alfa foi o,87 e não existiu indicação para eliminar qualquer dos itens. Com base no estudo destas amostras e de acordo com critérios psicométricos, pode-se afirmar que a PSS-10 tem uma boa consistência interna, não sendo evidente a necessidade de reformular ou eliminar nenhum dos seus itens. O coeficiente alfa $(\alpha=0,87)$ obtido para o total da amostra da população geral é, aliás, superior ao valor de 0,78 apresentado por Cohen e Williamson (1988) e por diversos autores (ver Quadro II). 
Quadro VII. Correlação item-total corrigida e coeficiente alfa de Cronbach da PSS-10, no grupo de saudáveis.

\begin{tabular}{l|l|l}
\hline \multirow{2}{*}{ Item n. $^{\circ}$} & \multicolumn{2}{|c}{$\begin{array}{c}\text { Homens e mulheres } \\
(n=745)\end{array}$} \\
\cline { 2 - 3 } & $\begin{array}{c}\text { Correlação item-total } \\
\text { corrigida }\end{array}$ & $\begin{array}{c}\text { Alfa de Cronbach } \\
\text { eliminando o item }\end{array}$ \\
\hline PSS1 & 0,517 & 0,868 \\
\hline PSS2 & 0,651 & 0,857 \\
\hline PSS3 & 0,612 & 0,861 \\
\hline PSS4 & 0,502 & 0,869 \\
\hline PSS5 & 0,652 & 0,858 \\
\hline PSS6 & 0,533 & 0,867 \\
\hline PSS7 & 0,484 & 0,870 \\
\hline PSS8 & 0,671 & 0,856 \\
\hline PSS9 & 0,621 & 0,860 \\
\hline PSS10 & 0,712 & 0,852 \\
\hline Correlação média inter-item & \multicolumn{2}{|c}{0,410} \\
\hline$\alpha$ & \multicolumn{2}{|c}{0,874} \\
\hline
\end{tabular}

\section{Médias e desvios-padrão}

Baseados na validação da PSS desenvolvida pelos autores (Cohen, Kamarck \& Mermelstein, 1983; Cohen \& Williamson, 1988), analisámos as pontuações médias $(M)$, o desvio-padrão (DP), os valores mínimos (mín.) / máximos (máx.) e as diferenças entre médias $(p)$, quer nas amostras da população geral quer nas amostras de participantes com doença (ver Quadro VIII). No primeiro caso, as amostras foram estratificadas por sexo, idade, estado civil, escolaridade e profissão.

- Sexo. A estratificação por sexo revelou que as mulheres $(M=16,6$; $D P=6,3)$, em relação aos homens $(M=13,4 ; D P=6,5)$, tiveram pontuações médias significativamente superiores $\left[t_{(743)}=-6,821 ; p<0,001\right]$. A tendência para as mulheres referirem níveis mais elevados na percepção do stresse é concordante com os resultados obtidos por Cohen e Williamson (1988), Hewitt, Flett e Mosher (1992) ou Remor (2006), embora, por exemplo, Roberti, Harrington e Storch (2006) não encontrem estas diferenças.

- Idade. Observou-se uma diferença significativa nas pontuações médias entre as mulheres jovens ( $17-25$ anos) e as adultas ( 26 - 65 anos), com as primeiras a pontuarem mais alto $\left.t_{(448)}=2,206 ; p<0,05\right]$. Embora os 
resultados não sejam conclusivos, eles parecem corroborar dados da literatura (Cohen \& Williamson, 1988), onde se refere que as pontuações na PSS diminuem à medida que a idade aumenta.

- Estado civil. Observaram-se, entre os vários estados civis, diferenças significativas nas pontuações médias obtidas na PSS-10 $\left[F_{(4,739)}=3,093\right.$; $p<0,05]$. Através de um teste post hoc (Scheffé), concluiu-se que os solteiros, em comparação aos casados, tinham maior percepção de stresse. Apesar deste dado não ser estranho à literatura (Cohen \& Williamson, 1988), importa salientar que, neste estudo, o estrato dos solteiros foi composto maioritariamente por mulheres $(n=268 ; 64,4 \%)$ e por estudantes $(n=219$; $52,8 \%$ ). Do grupo de estudantes, a maioria são mulheres ( $n=154 ; 70,3 \%$ ) que, como já se referiu, tendem a apresentar níveis de percepção do stresse mais elevados do que os homens.

- Escolaridade. Considerando que existiu uma diferença na pontuação média obtida na PSS-10, entre os graus de escolaridade $\left[F_{(3,741)}=3,171\right.$; $p<0,05]$, efectuou-se o teste Scheffé para identificar a razão desta diferença. Verificou-se, assim, que este efeito se deveu ao facto de as pessoas com frequência universitária (grau 1) apresentarem uma percepção do stresse significativamente maior do que os seus pares com dez a doze anos de escolaridade (grau 2). 
Quadro VIII. Pontuações médias, desvio-padrão, mínimo / máximo e diferenças de médias na PSS-10, em vários estratos.

\begin{tabular}{|c|c|c|c|c|c|c|c|}
\hline & Variáveis & $\mathrm{n}$ & $M$ & DP & mín. & máx. & $p$ \\
\hline \multirow{13}{*}{$\begin{array}{l}\overline{\frac{\pi}{2}} \\
\frac{0}{00} \\
0 \\
\frac{0}{0} \\
\frac{\pi}{2} \\
\frac{0}{0} \\
0\end{array}$} & Sexo & & & & & & \\
\hline & Homens & 295 & 13,4 & 6,5 & $\mathrm{O}$ & 35 & $* * *$ \\
\hline & Mulheres & 450 & 16,6 & 6,3 & O & 34 & \\
\hline & Jovens (17-25) & & & & & & \\
\hline & Homens & 93 & 14,3 & 6,1 & 3 & 28 & $* * *$ \\
\hline & Mulheres & 198 & 17,4 & 6,5 & o & 34 & \\
\hline & Adultos (26-65) & & & & & & \\
\hline & Homens & 202 & 12,9 & 6,6 & o & 35 & $* * *$ \\
\hline & Mulheres & 252 & 16,0 & 6,2 & o & 32 & \\
\hline & \begin{tabular}{|l|} 
Estado civil \\
Solteiro \\
Casado \\
União facto \\
Divorciado \\
Viúvo \\
\end{tabular} & $\begin{array}{c}408 \\
286 \\
13 \\
31 \\
6 \\
\end{array}$ & $\begin{array}{r}16,1 \\
14,4 \\
15,5 \\
15,3 \\
12,5\end{array}$ & $\begin{array}{l}6,4 \\
6,6 \\
8,2 \\
7,2 \\
6,0 \\
\end{array}$ & $\begin{array}{l}0 \\
0 \\
6 \\
0 \\
4\end{array}$ & $\begin{array}{l}34 \\
35 \\
31 \\
26 \\
19\end{array}$ & $*$ \\
\hline & $\begin{array}{l}\text { Escolaridade } \dagger \\
\text { Grau } 1 \text { (> } 12 \text { anos) } \\
\text { Grau } 2 \text { (10 - } 12 \text { anos) } \\
\text { Grau } 3 \text { ( } 8 \text { - } 9 \text { anos) } \\
\text { Grau } 4 \text { ( } 4-6 \text { anos) } \\
\end{array}$ & $\begin{array}{c}532 \\
109 \\
55 \\
49 \\
\end{array}$ & $\begin{array}{l}15,6 \\
13,6 \\
16,3 \\
15,1 \\
\end{array}$ & $\begin{array}{l}6,5 \\
6,5 \\
6,7 \\
7,6\end{array}$ & $\begin{array}{l}0 \\
0 \\
1 \\
1\end{array}$ & $\begin{array}{l}35 \\
31 \\
31 \\
32\end{array}$ & $*$ \\
\hline & $\begin{array}{l}\text { Profissão † } \\
\text { Grau } 1 \text { (ex. Director) } \\
\text { Grau } 2 \text { (ex. Sub-director) } \\
\text { Grau } 3 \text { (ex. Capataz) } \\
\text { Grau } 4 \text { (ex. Polícia) } \\
\text { Grau } 5 \text { (ex. Jornaleiro) } \\
\text { Estudantes }\end{array}$ & $\begin{array}{c}204 \\
42 \\
189 \\
64 \\
23 \\
221 \\
\end{array}$ & $\begin{array}{r}14,2 \\
14,5 \\
15,1 \\
15,9 \\
16,5 \\
16,4\end{array}$ & $\begin{array}{l}6,0 \\
6,2 \\
7,1 \\
7,2 \\
6,9 \\
6,4\end{array}$ & $\begin{array}{l}0 \\
5 \\
0 \\
1 \\
1 \\
0\end{array}$ & $\begin{array}{l}30 \\
31 \\
34 \\
35 \\
32 \\
31\end{array}$ & $*$ \\
\hline & $\begin{array}{l}\text { Hábitos tabágicos } \\
\text { Não-fumador } \\
\text { Ex-fumador } \\
\text { Fumador }\end{array}$ & $\begin{array}{c}409 \\
135 \\
200 \\
\end{array}$ & $\begin{array}{r}15,1 \\
14,6 \\
16,3\end{array}$ & $\begin{array}{l}6,6 \\
5,8 \\
6,9\end{array}$ & $\begin{array}{l}0 \\
1 \\
0\end{array}$ & $\begin{array}{l}34 \\
28 \\
35\end{array}$ & $*$ \\
\hline \multirow{4}{*}{ 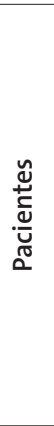 } & $\begin{array}{l}\text { DAC } \\
\text { Homens } \\
\text { Mulheres } \\
\end{array}$ & $\begin{array}{l}41 \\
10 \\
\end{array}$ & $\begin{array}{r}13,4 \\
20,8 \\
\end{array}$ & $\begin{array}{l}7,9 \\
9,8\end{array}$ & $\begin{array}{l}0 \\
6 \\
\end{array}$ & $\begin{array}{l}29 \\
39\end{array}$ & $*$ \\
\hline & $\begin{array}{l}\text { DPOC } \\
\text { Homens } \\
\text { Mulheres } \\
\end{array}$ & $\begin{array}{c}44 \\
6 \\
\end{array}$ & $\begin{array}{l}16,1 \\
17,0\end{array}$ & $\begin{array}{l}8,5 \\
5,0\end{array}$ & $\begin{array}{c}0 \\
10 \\
\end{array}$ & $\begin{array}{l}36 \\
24 \\
\end{array}$ & NS \\
\hline & \begin{tabular}{|l|} 
HDD \\
Homens \\
Mulheres \\
\end{tabular} & $\begin{array}{l}15 \\
12 \\
\end{array}$ & $\begin{array}{l}12,4 \\
18,3 \\
\end{array}$ & $\begin{array}{l}4,5 \\
5,6 \\
\end{array}$ & $\begin{array}{c}3 \\
10 \\
\end{array}$ & $\begin{array}{l}19 \\
31\end{array}$ & $* *$ \\
\hline & $\begin{array}{l}\text { Ansiosos } \\
\text { Homens } \\
\text { Mulheres }\end{array}$ & $\begin{array}{l}15 \\
20\end{array}$ & $\begin{array}{l}24,2 \\
24,1\end{array}$ & $\begin{array}{l}8,2 \\
8,1\end{array}$ & $\begin{array}{l}11 \\
11\end{array}$ & $\begin{array}{l}40 \\
37\end{array}$ & NS \\
\hline
\end{tabular}

DAC = Doença das artérias coronárias; $D P O C=$ Doença pulmonar obstrutiva crónica; HDD = Hipertensão arterial, dislipidémia ou diabetes; NS = Não significativo; ${ }^{*} \mathrm{p}<0,05 ;{ }^{* *} \mathrm{p}<0,01 ;{ }^{* * *} \mathrm{p}<0,001 ;$ † Classificação baseada no Graffar (1956) modificado. 
- Profissão. A estratificação em função da profissão revela um resultado interessante, com a percepção do stresse a diminuir à medida que a categoria profissional é mais elevada. As diferenças entre as pontuações médias na PSS-10 foram significativas $\left[F_{(5,737)}=2,940 ; p<0,05\right]$ e pelo teste Scheffé, concluiu-se que este resultado se deveu às médias dos profissionais de grau 1, em comparação aos estudantes. Como se sabe, os estudantes estão sujeitos a pressões relacionadas com o seu desempenho académico e, neste estudo, trata-se de um grupo com elevada percentagem de mulheres (70,3\%).

- Hábitos tabágicos. Podemos observar que os fumadores $(M=16,3 ; D P=6,9)$ obtiveram pontuações significativamente $\left[F_{(2,741)}=3,047 ; p<0,05\right]$ mais elevadas do que os ex-fumadores $(M=14,6 ; D P=5,8)$ e os não fumadores ( $M$ $=15,1 ; D P=6,6)$. Esta diferença é concordante com os resultados de Cohen e Williamson (1988) e parece confirmar que os fumadores apresentam níveis mais elevados de stresse (Parrott, 1995, 1999, 2000).

Considerando que as amostras de participantes com diagnóstico têm menor dimensão, apenas se procedeu à estratificação por sexo. Em cada uma das amostras, por seu turno, calcularam-se as pontuações médias ( $M)$, o desvio-padrão (DP), os valores mínimos (mín.) / máximos (máx.) e as diferenças entre médias ( $p$ ). Atendendo às diferenças na natureza dos vários quadros clínicos, não se assumiu existir um grupo homogéneo de pacientes, com excepção óbvia das amostras 9 e 10 (doentes ansiosos). Como o estrato das mulheres apresentava um número de sujeitos inferior a trinta, recorreu-se ao teste não-paramétrico Mann-Whitney $U$. A reduzida dimensão destas amostras justificam prudência adicional na interpretação e extrapolação dos resultados:

- Doentes das artérias coronárias (DAC). Na amostra de pacientes coronários, os homens $(M=13,4 ; D P=7,9)$, comparativamente às mulheres $(M=20,8$; $D P=9,8)$, apresentaram valores médios na percepção de stresse significativamente inferiores $(U=112,000 ; p<0,05)$. Este resultado, sugere existir maior susceptibilidade feminina ao stresse.

- Doentes pulmonares obstrutivos crónicos (DPOC). No caso dos doentes pulmonares, os homens $(M=16,1 ; D P=8,5)$ e as mulheres $(M=17 ; D P=5)$ obtiveram pontuações médias semelhantes.

- Hipertensão arterial, dislipidémia e diabetes (HDD). Os cálculos efectuados apontam para uma média nos homens $(M=12,4 ; D P=4,5)$ inferior ao das mulheres $(M=18,3 ; D P=5,6)$, revestindo-se esta diferença de significância estatística $(U=35,000 ; p<0,01)$. Note-se, porém, que a reduzida dimensão da amostra $(n=27)$ exige especial cautela na análise deste resultado. 
- Perturbações da ansiedade. De todas as amostras estudadas (amostras 1 a 8), são os homens $(M=24,2 ; D P=8,2)$ e as mulheres $(M=24,1 ; D P=8,1)$, com diagnóstico de perturbação ansiosa, aqueles que revelaram pontuações mais elevadas na percepção do stresse. Comparando as pontuações médias das amostras da população geral $(M=15,3 ; D P=6,6)$ e das pessoas com perturbação ansiosa $(M=24,1 ; D P=8,1)$, observou-se uma diferença significativa $\left[t_{(778)}=-7,663 ; p<0,001\right]$. Este resultado não é inesperado, dado que estas sindromas envolvem sensações de tensão, ansiedade, insegurança, medo, cognições catastróficas, baixa da auto-estima e fraco sentimento de competência pessoal (APA, 2002; Beck, Emery \& Greenberg, 1985).

\section{Distribuição das pontuações brutas}

Com o objectivo de adquirir uma perspectiva geral acerca da distribuição das pontuações médias brutas obtidas na PSS-10, estabeleceu-se uma equivalência entre estas pontuações e o respectivo percentil. Para o efeito, consideraram-se ambos os grupos ( $n=912)$, sem e com doença. Atendendo, porém, às diferenças encontradas entre homens $(n=414)$ e mulheres $(n=498)$, estratificou-se por sexo. Nos homens, as pontuações médias brutas de 13,9 $(D P=7,2)$ situaram-se no percentil 50, enquanto as mulheres do mesmo percentil obtiveram pontuações de $17,1(D P=6,6)$. Tendo como referência os resultados que os homens e as mulheres com perturbações da ansiedade alcançaram $(M=24 ; D P=8)$, estabeleceu-se que as notas acima do percentil 80 constituem indicadores significativos de patologia (ver Quadro IX).

Quadro IX. Equivalência entre as notas brutas da PSS-10 e os respectivos percentis, estratificado por sexo.

\begin{tabular}{|c|c|c|}
\hline Percentil & $\begin{array}{l}\text { Homens } \\
(n=414)\end{array}$ & $\begin{array}{l}\text { Mulheres } \\
(n=498)\end{array}$ \\
\hline 10 & 5,5 & 9,0 \\
\hline 20 & 8,0 & 11,0 \\
\hline 25 & 9,0 & 12,0 \\
\hline 30 & 10,0 & 13,7 \\
\hline 40 & 12,0 & 15,0 \\
\hline 50 & 13,0 & 17,0 \\
\hline 60 & 15,0 & 18,0 \\
\hline 70 & 17,0 & 20,0 \\
\hline 75 & 18,0 & 21,0 \\
\hline $80+$ & 20,0 & 22,0 \\
\hline 90 & 24,0 & 26,0 \\
\hline
\end{tabular}

† Ponto de corte para pontuações patológicas na PSS-10. 


\section{Correlação com outros indicadores e medidas de saúde}

Tendo em conta a diferença média nas pontuações da PSS-10, obtida pelos homens e mulheres saudáveis, decidiu-se apresentar valores totais estratificados por sexo. Partiu-se do princípio de que o aumento na percepção de stresse estaria associado à correspondente subida das pontuações obtidas em outras medidas e indicadores relacionados com a saúde (hábitos tabágicos, BDI ${ }^{\circ}$, STAI ${ }^{\circ}$-Y2 e perturbações ansiosas). Inversamente, seria de esperar uma associação negativa ou a ausência de associação entre as pontuações da PSS-10 e uma medida de auto-eficácia (SASE-12), aplicada a um grupo de fumadores. A fim de avaliar a equivalência entre a PSS-10 e a versão de quatro itens (PSS-4), estudou-se também a sua inter-correlação.

Tomando em consideração o conjunto de homens e mulheres, verifica-se que a PSS-10 mantém correlações significativas, positivas e moderadas com o STAI ${ }^{\circ}-Y_{2}$ $\left[r_{(730)}=0,675 ; p<0,01\right]$ e o $B D I^{\circ}\left[r_{(739)}=0,530 ; p<0,01\right]$. Por seu turno, os fumadores, em comparação $\left[t_{(778)}=-7,663 ; p<0,001\right]$ com as pessoas sem hábitos tabágicos (ex-fumadores e não fumadores) e os ansiosos em comparação com as pessoas sem perturbação da ansiedade $\left[t_{(778)}=-7,663 ; p<0,001\right]$, obtiveram pontuações médias na PSS-10 significativamente mais elevadas. Em resumo, a PSS-10 apresentou uma convergência bastante satisfatória com outras medidas que avaliam dimensões próximas, designadamente sensações de pressão ou tensão emocional. Da mesma forma, o tabagismo e as perturbações da ansiedade, associaram-se ao aumento da percepção de stresse.

Por seu turno, registou-se uma correlação sem significância estatística $\left[r_{(197)}=-0,074 ; N S\right]$, entre a pontuação da PSS-10 e a percepção de auto-eficácia em fumadores (SASE-12). Este resultado é compreensível, na medida em que o sentimento de competência pessoal tende a não apresentar relação com a percepção de stresse, a depressão ou a ansiedade. Em relação à equivalência entre a PSS-10 e a PSS-4, registaram-se correlações significativas, positivas e fortes $\left[r_{(744)}=0,923 ; p<0,001\right]$, o que sugere a possibilidade de usar, com eficácia, a PSS-4. No seu conjunto, estes dados corroboram premissas da comunidade científica e clínica, dando bons indicadores sobre a validade da PSS-10 e da PSS-4 (ver Quadro X). 
Quadro X. Coeficientes de correlação Pearson entre a pontuação da PSS-10 e outras medidas de saúde, estratificadas por sexo.

\begin{tabular}{|c|c|c|c|}
\hline \multirow[b]{2}{*}{ Instrumentos } & \multicolumn{3}{|c|}{ PSS-10 } \\
\hline & $\begin{array}{l}\text { Homens } \\
\text { (n) }\end{array}$ & $\begin{array}{l}\text { Mulheres } \\
\text { (n) }\end{array}$ & $\begin{array}{l}\mathrm{H} \text { e } \mathrm{M} \\
\text { (n) }\end{array}$ \\
\hline $\begin{array}{l}\mathrm{BDI}^{\oplus \dagger} \\
\text { Depressão }\end{array}$ & $\begin{array}{c}0,489^{* *} \\
(293)\end{array}$ & $\begin{array}{l}0,528^{* *} \\
(446)\end{array}$ & $\begin{array}{l}0,530^{* *} \\
(739)\end{array}$ \\
\hline $\begin{array}{l}\text { STAI }^{\circledR}-\mathbf{Y}_{\mathbf{2}} \ddagger \\
\text { Traço de ansiedade }\end{array}$ & $\begin{array}{c}0,680^{* *} \\
(288)\end{array}$ & $\begin{array}{c}0,645^{* *} \\
(442)\end{array}$ & $\begin{array}{l}0,675^{* *} \\
(730)\end{array}$ \\
\hline $\begin{array}{l}\text { SASE-12 } \\
\text { Auto-eficácia }\end{array}$ & $\begin{array}{c}-0,105 \text { NS } \\
(85)\end{array}$ & $\begin{array}{c}0,002 \text { NS } \\
(112)\end{array}$ & $\begin{array}{c}-0,074 \text { NS } \\
(197)\end{array}$ \\
\hline $\begin{array}{l}\text { PSS-4 } \\
\text { Stresse }\end{array}$ & $\begin{array}{c}0,9133^{* * *} \\
(294)\end{array}$ & $\begin{array}{l}0,926^{* * *} \\
(450)\end{array}$ & $\begin{array}{c}0,923^{* * *} \\
(744)\end{array}$ \\
\hline
\end{tabular}

$\mathrm{H}$ e $\mathrm{M}=$ Homens e mulheres; $\mathrm{NS}=$ Não significativo; ${ }^{* *} \mathrm{p}<0,01 ;{ }^{* * *} \mathrm{p}<0,001 ; \dagger \mathrm{O}$ cálculo da pontuação total do BDI ${ }^{\otimes}$ exclui os itens 1, 2, 4, 5, 6, 8, 11, 12, 16, 18, 19 e 20; $¥ 0$ cálculo da pontuação total do STAI ${ }^{\oplus}-Y_{2}$ exclui os itens 2, 4, 5, 8, 12, 13, 14 e 15. Nos instrumentos utilizados, a exclusão de itens está de acordo com o estudo das qualidades psicométricas previamente realizado.

\section{Conclusões}

A versão de dez itens da Perceived Stress Scale - PSS-10 (Cohen, Kamarck \& Mermelstein, 1983) constitui uma medida global que avalia até que ponto os acontecimentos de vida são percepcionados como indutores de stresse. Nos estudos efectuados na população portuguesa, com amostras da população geral ou com condições de doença física e perturbação ansiosa, obtiveram-se indicadores bastante positivos no que concerne às qualidades psicométricas da PSS-10. Através da análise dos componentes principais, com rotação Varimax, extraiu-se um único componente. A consistência interna para a população geral apresentou um coeficiente alfa elevado, aliás superior ao obtido pelo autor. Com base em critérios estatísticos, não se encontraram itens com baixa saturação factorial ou cuja eliminação contribuísse para melhorar o alfa de Cronbach. Em termos da distribuição da percepção do stresse, observaram-se níveis médios significativamente mais elevados nas mulheres, nos jovens, nos solteiros, nas pessoas com habilitações universitárias, no grupo de estudantes, nos fumadores e nos ansiosos. Com vista a identificar níveis patológicos na percepção de stresse, estabeleceu-se um valor de corte para pontuações superiores ao percentil 80 .

Relativamente à associação entre a percepção do stresse e outros indicadores ou medidas de saúde, obtiveram-se correlações positivas com a depressão, a ansiedade, o tabagismo e o diagnóstico de perturbação ansiosa. Estes resultados parecem estar de acordo com a literatura, pois a percepção de stresse tende a associar-se à activação 
de sistemas e estados emocionais negativos, designadamente ansiosos e depressivos (Brown, 1998; Dohrenwend et al., 1995; Faravelli, Paterniti e Servi, 1997; Goldberg \& Huxley, 1996). Inversamente, não se registou correlação entre a pontuação da PSS e a auto-eficácia para não fumar em situações de tentação. Com efeito, sabe-se que o sentimento de auto-eficácia pode coexistir quer com níveis elevados de percepção do stresse, quando, por exemplo, alguém responde a um teste difícil para o qual se preparou quer com níveis baixos de stresse, por exemplo, ao desempenhar tarefas complexas que se automatizaram através de uma aprendizagem profunda.

De uma forma geral, a versão portuguesa da PSS-10 mostrou-se robusta, de fácil compreensão, preenchimento rápido e simples de cotar. A versão de quatro itens pode revelar igual eficácia na avaliação do stresse. Por seu turno, sendo a instrução desta escala relativa à frequência com que a pessoa se sentiu durante o último mês, deve-se concluir que a PSS se destina a avaliar estados e não padrões estáveis de percepção do stresse. Note-se, porém, que devido à elevada validade facial da PSS é fácil responder segundo um estereótipo social desejável, o que constitui um aspecto menos favorável na utilização da escala.

O facto de se tratar de uma medida global do stresse torna a PSS particularmente adaptável a diferentes contextos de saúde, doença física ou perturbação emocional, designadamente nas situações em que seja útil fazer avaliações antes e depois de uma modificação comportamental ou intervenção cirúrgica, na recuperação de quadros agudos de doença, no acompanhamento do tratamento de toxicodependentes, na avaliação de familiares de doentes, em contextos escolares, de aprendizagem e períodos de exame ou em âmbitos organizacionais e empresariais. Esta abrangência implica, contudo, o recurso a outros instrumentos sempre que seja necessária maior especificidade na avaliação do stresse.

\section{Referências bibliográficas}

Adrienne, S. \& Barna, K. (2006). Characteristics of the Hungarian version of the Perceived Stress Scale (PSS). Mentalhigiene es Pszichoszomatika, 7, 203-216.

Almeida, L.; Simões, M.; Machado, C. \& Gonçalves, M. (2008). Avaliação psicologia: Instrumentos validados para a população portuguesa (Vol. II). Coimbra: Quarteto.

Anastasi, A. (1977). Testes psicológicos ( $2^{\circ}$ Edição). São Paulo: Editora Pedagógica e Universitária, Lda.

APA - American Psychiatric Association (2002). DSM-IV-TR. Manual de diagnóstico e estatís-

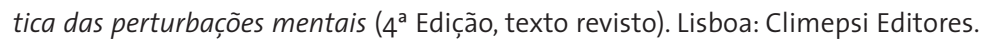

Beck, A.; Emery, G. \& Greenberg, R. (1985). Anxiety disorders and phobias. A cognitive perspective. USA: Basic Books.

Beck, A.; Rush, A.; Shaw, B. \& Emery, G. (1979). Cognitive therapy of depression. New York: The Guilford Press. 
Bernard, C. (1879). Leçons sur les phénomènes de la vie commune aux animaux et aux végétaux (Vol. 2). Paris: Baillières.

Bernard, C. (1978). Introdução à medicina experimental. Lisboa: Guimarães \& C. ${ }^{a}$ Editores.

Breznitz, S. \& Goldberg, L. (1993). Stress research at a crossroads. L. Goldberg \& S. Breznitz (Editors). Handbook of stress. Theoretical and clinical aspects (pp. 3-6). New York: The Free Press.

Brown, G. (1998). Loss and depressive disorders. B. Dohrenwend (Editor). Adversity, stress and psychopathology (pp. 358-370). New York: Oxford University Press.

Canon, W. (1939). The wisdom of the body. New York: Norton.

Cohen, S.; Kamarck, T. \& Mermelstein, R. (1983). A global measure of perceived stress. Journal of Health and Social Behavior, 24, 385-396.

Cohen, S. \& Williamson, G. (1988). Perceived stress in a probability sample of the United Sates. S. Spacapan \& S. Oskamp (Editors). The social psychology of health (pp. 31-67). Newbury Park: Sage.

Cole, S. (1999). Assessment of differential item functioning in the Perceived Stress Scale-10. Journal of Epidemiology and Community Health, 53, 319-320.

Derogatis, L. \& Coons, H. (1993). Self-report measures of stress. L. Goldberg \& S. Breznitz (Editors). Handbook of stress. Theoretical and clinical aspects (pp. 200-233). New York: The Free Press.

DiClemente, C. (1981). Self-efficacy and smoking cessation maintenance: A preliminary report. Cognitive Therapy and Research, 5, 175-187.

Dohrenwend, B.; Shrout, P.; Link, B.; Skodol, A. \& Stueve, A. (1995). Life events and other possible risk factors for episodes of schizophrenia and major depression: A casecontrol study. C. Mazure (Editor). Does stress cause psychiatric illness? (pp. 43-65). Washington: American Psychiatric Press.

Faravelli, C.; Paterniti, S. \& Servi, P. (1997). Stressful life events and panic disorder. T. Miller (Editor). Clinical disorder and stressful life events (pp. 143-170). Madison: International Universities Press, Inc.

Goldberg, D. \& Huxley, P. (1996). Perturbações mentais comuns. Um modelo bio-social. Lisboa: Climepsi Editores.

Golden-Kreutz, D.; Browne, M.; Frierson, G. \& Anderson, B. (2004) Assessing stress in cancer patients: A second-order factor analysis model for the Perceived Stress Scale. Assessment, 11, 216-223.

Gonçalves, M.; Simões, M.; Almeida, L. \& Machado, C. (2006). Avaliação psicologia: Instrumentos validados para a população portuguesa (Vol. I). Coimbra: Quarteto.

Graffar, M. (1956). Une méthode de classification sociale d'échantillons de population. Courrier, 6, 455-459.

Guillet, L. \& Hermand, D. (2006). Critique de la mesure du stress. L'Année Psychologique, $106,129-164$

Hewitt, P.; Flett, G. \& Mosher, S. (1992). The Perceived Stress Scale: Factor structure and relation to depression symptoms in a psychiatric sample. Journal of Psychopathology and Behavioral Assessment, 14, 247-257.

Holmes, T. \& Rahe, R. (1967). The Social Readjustment Scale. Journal of Psychosomatic Research, $11,213-218$

Lazarus, R. (1999). Stress and emotion: A new synthesis. New York: Springer Publishing Co.

Lazarus, R. (1991). Emotion and adaptation. New York: Oxford University Press.

Lazarus, R. \& Folkman, S. (1984). Stress, appraisal, and coping. New York: Springer Publishing Company. 
Luft, C.; Sanches, S.; Mazo, G. \& Andrade, A. (2007). Versão brasileira da Escala de Estresse Percebido: Tradução e validação para idosos. Revista de Saúde Pública, 41, 606-615.

Meyer, A. (1930). Reminiscences and prospects at the opening of the New York Psychiatric Institute and Hospital. Psychiatric Quarterly, 4, 25-34.

Mimura, C. \& Griffiths, P. (2004). A Japanese version of the Perceived Stress Scale: Translation and preliminary test. International Journal of Nursing Studies, 41, 379-385.

Nunnally, J. \& Bernstein, I. (1994). Psychometric theory (3 ${ }^{\text {rd }}$ Edition). New York: McGraw-Hill, Inc. Parrott, A. (2000). Cigarette smoking does cause stress. American Psychologist, October, 1159-1160. Parrott, A. (1999). Does cigarette smoking cause stress? American Psychologist, 54, 817-820.

Parrott, A. (1995). Smoking cessation leads to reduced stress, but why? International Journal of the Addictions, 30, 1509-1516.

Pestana, M. \& Gageiro, J. (2005). Análise de dados para ciências sociais. A complementaridade do SPSS ( $4^{\mathrm{a}}$ edição). Lisboa: Edições Sílabo.

Quick, J.; Quick, J. \& Gavin, J. (2000). Stress: Measurement. A. Kazdin (Editor). Encyclopedia of psychology (pp. 484-487). Washington: American Psychological Association, Oxford University Press.

Ramírez, M. \& Hernández, R. (2007). Factor structure of the Perceived Stress Scale (PSS) in a sample from Mexico. The Spanish Journal of Psychology, 10, 199-206.

Remor, E. (2006). Psychometric properties of a European Spanish version of the Perceived Stress Scale (PSS). The Spanish Journal of Psychology, 9, 86-93.

Remor, E. \& Carrobles, J. (2001). Version Espanola de la Escala de Estress Percebido (PSS-14): Estudio psicometrico en una muestra $\mathrm{VIH}+$. Ansiedad y Estress, 7, 195-201.

Ribeiro, J. (2007). Avaliação em psicologia da saúde: Instrumentos publicados em português. Coimbra: Quarteto.

Roberti, J.; Harrington. L. \& Storch, E. (2006). Further psychometric support for the 10-item version of the Perceived Stress Scale. Journal of College Counseling, 9, 135-147.

Selye, H. (1936). A syndrome produced by diverse nocuous agents. Nature, 138, 32

Sharp, L.; Kimmel, L.; Kee, R.; Saltoun, C. \& Chang, C. (2007). Assessing the Perceived Stress Scale for African American adults with asthma and low literacy. Journal of Asthma, 44, 311-316.

Shigetoshi, l.; Yoshiki, T.; Shin, F. \& Michiot, H. (2002). The development of the Japanese version of the Perceived Stress Scale. Japanese Journal of Psychosomatic Medicine, 42, 459-466.

Silva, D. \& Campos, R. (1999). Alguns dados normativos do Inventário de Estado-Traço de Ansiedade - forma Y (STAI-Y) de Spielberger, para a população portuguesa. Revista Portuguesa de Psicologia, 33, 71-89.

Silva, D. \& Correia, S. (s/d). Versão e preparação portuguesa do State-Trait Anxiety Inventory Form $Y\left(S T A l^{\circ}\right)$. Tradução e preparação autorizadas no âmbito do estudo de adaptação.

Simões, M.; Machado, C.; Gonçalves, M. \& Almeida, L. (2007). Avaliação Psicologia: Instrumentos validados para a população portuguesa (Vol. III). Coimbra: Quarteto.

Spielberger, C. (1983). State-Trait Anxiety Inventory (Form Y). Palo Alto: Mind Garden.

Sumi, K. (2006). Reliability and validity of the Japanese version of the Perceived Stress Scale. Japanese Journal of Health Psychology, 19, 44-53.

Trigo, M. (2007). Cigarros, tabaco e nicotina: Factores biocomportamentais associados ao tabagismo. Dissertação de Doutoramento apresentada à Faculdade de Psicologia e de Ciências da Educação da Universidade de Lisboa.

Trigo, M.; Canudo, N.; Rocha, E. \& Silva, D. (2007). Entrevista estruturada para avaliação de hábitos tabágicos. M. Trigo. Cigarros, tabaco e nicotina: Factores biocomportamentais 
associados ao tabagismo. Dissertação de Doutoramento apresentada à Faculdade de Psicologia e de Ciências da Educação da Universidade de Lisboa.

Trigo, M. \& Silva, D. (2003). Versão e preparação portuguesa do Perceived Stress Scale - 10 (PSS-10). Tradução e utilização autorizadas pelo autor.

Trigo, M. \& Silva, D. (2003a). Versão e preparação portuguesa do Beck Depression Inventory $\left(B D I^{\circ}\right)$. Tradução e reprodução autorizada pela Harcourt Assessment, Inc.

Trigo, M. \& Silva, D. (2003b). Versão e preparação portuguesa do Smoking Abstinence Self-Efficacy (SASE-12). Tradução e utilização autorizadas.

WHO - World Health Organization (2005). International statistical classification of diseases and related health problems - ICD-10 ( $2^{\text {nd }}$ Edition). Geneva: WHO.

\section{Anexo}

\section{Instruções e itens traduzidos da Perceived Stress Scale $e^{13}$}

Para cada questão, pedimos que indique com que frequência se sentiu ou pensou de determinada maneira, durante o último mês. Apesar de algumas perguntas serem parecidas, existem diferenças entre elas e deve responder a cada uma como perguntas separadas. Responda de forma rápida e espontânea. Para cada questão, escolha a alternativa que melhor se ajusta à sua situação.

o - Nunca. 1 - Quase nunca. 2 - Algumas vezes. 3 - Frequentemente. 4 - Muito frequentemente.

1. No último mês, com que frequência esteve preocupado(a) por causa de alguma coisa que aconteceu inesperadamente?

2. No último mês, com que frequência se sentiu incapaz de controlar as coisas importantes da sua vida?

3. No último mês, com que frequência se sentiu nervoso(a) e em stresse?

$4^{\dagger}$. No último mês, com que frequência sentiu confiança na sua capacidade para enfrentar os seus problemas pessoais?

5t. No último mês, com que frequência sentiu que as coisas estavam a correr à sua maneira?

6. No último mês, com que frequência sentiu que não aguentava com as coisas todas que tinha para fazer?

$7 \dagger$. No último mês, com que frequência foi capaz de controlar as suas irritações?

$8+$. No último mês, com que frequência sentiu ter tudo sob controlo?

9. No último mês, com que frequência se sentiu furioso(a) por coisas que ultrapassaram o seu controlo?

10. No último mês, com que frequência sentiu que as dificuldades se estavam a acumular tanto que não as conseguia ultrapassar?

† Itens cotados de forma inversa.

13 Perceived Stress Scale (Cohen, Kamarck \& Mermelstein, 1983). Escala do Stresse Percepcionado. Versão portuguesa preparada por Miguel Trigo e Danilo Silva (2003). Tradução autorizada pelo autor. 


\section{Psychometric proprieties of the Perceived Stress Scale (PSS) in Portuguese population.}

Objective: To study the psychometric properties of the ten item Perceived Stress Scale-10 (PSS-10), in non-patient and patient samples of the Portuguese population. Sample: Five samples of healthy persons $(n=762)$, and five samples of patients with physical chronic disease or anxiety disorder $(n=201)$.

Measurements: Social-demographic data, smoking habits, Beck Depression Inventory ${ }^{\circ}\left(\mathrm{BDI}^{\circ}\right)$, PSS-10, State-Trait Anxiety Inventory ${ }^{\circ}\left(\mathrm{STAl}^{\circ}{ }^{\circ} \mathrm{Y}_{2}\right)$, and Smoking Abstinence Self-Efficacy (SASE-12).

Results: Using a principal component analysis, it was extracted one factor, that explained $47,4 \%$ of the variance. The Cronbach alpha was 0,874 , and meaningful differences in stress perception were found between several population strata. There are moderate correlations between PSS-10, the $\mathrm{BDI}^{\circ}$, and the STAI ${ }^{\circ}-\mathrm{Y}_{2}$, and absence of association with SASE-12. Scores above percentile 80 are pathological. Conclusions: PSS-10 reveals good psychometric properties. Administration and scoring is simple, and it is a valid assessment of stress, in physical disease or psychopathological contexts.

KEY-WORDS: Perceived Stress Scale. Stress. Escala do Stresse Percepcionado. Psychometric proprieties.

\section{Étude des propriétés psychométriques de l'échelle Perceived Stress Scale} (PSS) dans la population portugaise.

Objectif: Étudier les propriétés psychométriques de la version portugaise de l'échelle Perceived Stress Scale-10 (PSS-10).

Échantillon: Cinq échantillons de la population générale $(n=762)$ et cinq échantillons de personnes avec maladie physique ou trouble anxieux $(n=201)$.

Mesures: Données sociodémographiques, dépendance tabagique, Beck Depression Inventory ${ }^{\circ}\left(\mathrm{BDI}^{\circ}\right)$, PSS-10, State-Trait Anxiety Inventory ${ }^{\circ}$ (STAI $\left.{ }^{\circ}-\mathrm{Y}_{2}\right)$ et Smoking Abstinence Self-Efficacy (SASE-12).

Résultats: En utilisant l'analyse en composants principaux, on a retenu un facteur qui explique $47,4 \%$ de la variance. Lalfa de Cronbach a atteint o,874 et on a trouvé des différences significatives dans la perception du stress entre les différentes strates de la population. II y a des corrélations modérées entre le PSS-10, le BDI et le STAI ${ }^{\circ} \mathrm{Y}_{2}$ et aucune association avec le SASE-12. On considère comme indicateurs significatifs de pathologie les ponctuations brutes au-dessus de $80 \%$.

Conclusions: Le PSS-10 présente de bonnes qualités psychométriques. Ayant une application et cotation facile, c'est un instrument valable pour la mesure du stress, en cas de maladies psysiques ou de conditions psychopathologiques.

MOTS-CLÉS: Perceived Stress Scale. Escala do Stresse Percepcionado. Stress. Propriétés psychométriques. 